\title{
The importance of pattern information for the resolution of depth-ambiguous apparent motion
}

\author{
D. G. WHITE, PETER WENDEROTH, and I. S. CURTHOYS \\ Sydney University, Sydney 2006, New South Wales, Australia
}

\begin{abstract}
A single apparent motion display can result in the perception of a rigid three-dimensional motion or a plastic, two-dimensional motion. Previous studies have found that the principal determinant of the perceptual outcome is the temporal properties of the apparent motion stimulus. Here it is shown that the form properties of the stimulus are another determinant and that, in some situations, they may become a more powerful determinant than the temporal properties.
\end{abstract}

When certain types of figures are used in an apparent motion display, the resulting percept is depth ambiguous. That is, the phenomenal object can appear to slide in the frontoparallel plane or it can appear to rotate in depth towards or away from the observer. The sliding (two-dimensional) percept is characterized by bending and twisting of the contours of the phenomenal object so that during the motion one shape is deformed into the other. When the object appears to rotate (move three dimensionally), the phenomenal object remains rigid as it successively changes orientation, so that the percept is of the one shape being rotated from one position to another.

The usual technique for producing depth-ambiguous apparent motion (DAAM) is to take a figure which can be said to be pointing in, or facing, some particular direction and to pair it with the same figure pointing in a different direction. For example, Orlansky (1940) used arrows pointing in different directions or curved lines that faced in different directions, while Kolers and Pomerantz (1971) used a trapezoid. Apart from the property of "direction," the shapes used in these experiments seem to have been chosen randomly, and there is currently no way of predicting the depth ambiguity of a given stimulus array. One aim of the present study was to explore the importance of some aspects of the form itself in determining DAAM.

Using a trapezoidal stimulus, Kolers and Pomerantz (1971) found that at short interstimulus intervals (ISI) the plastic, two-dimensional type of motion was most often reported, whereas at longer ISIs reports of rigid three-dimensional motion predominated.

This report is based on a portion of a dissertation for the degree of Master of Science at Sydney University. Thanks are extended to Drs. P. M. Wenderoth and H. Beh for the loan of equipment that was supplied with the assistance of Australian Research Grants Committee Grant A74/15177. Reprint requests should be addressed to the senior author, Sydney University, New South Wales 2006, Australia.
These results occurred for all orientations of the figures. The different orientations had little effect on the type of motion reported. Averaging over a number of experimental conditions, Kolers and Pomerantz found that the peak two-dimensional response occurred at an ISI of $50 \mathrm{msec}$ and the peak three-dimensional response occurred at an ISI of $260 \mathrm{msec}$. They concluded that ISI was the single most important variable to resolve the ambiguity in DAAM. If ISI is the principal determinant of the type of motion reported, then this phenomenon may be another instance of the principle that as the phenomenal separation of the two figures generating the motion is increased the ISI must also be increased to preserve "good" motion, that is, motion that is smooth, continuous, and compelling.

The most common explanation of DAAM (Attneave, 1974; Kolers \& Pomerantz, 1971; Orlansky, 1940) is that when the object appears to travel in depth the phenomenal path it follows is longer than the path it follows when it moves two-dimensionally. Since longer paths require more time if "good", motion is to be seen, three-dimensional motion will tend to occur at longer ISIs than two-dimensional motion. This idea is supported by Shepard and Judd (1976), who found that an illusion of rigid rotation can be produced by presenting, in alternation, perspective views of the same three-dimensional object in two orientations. The minimum cycle time required for the illusion increases as the angular difference between the orientations is increased.

The possibility remains that the apparent velocity of a phenomenal object moving in depth may be different from the velocity of that object moving two-dimensionally. The shapes themselves may also be important in determining the type of motion seen. Some evidence suggests that there are apparent motion displays which almost always move in depth (the socalled Neuhaus triangles, for example), whereas other displays rarely, if ever, produce three-dimensional motion (for example, when both shapes at the termini 
of the motion are identical). It is the contention of this paper that the stimulus configuration, that is, the shapes and their relationship to one another, is critically important in determining the type of motion that is seen.

\section{EXPERIMENT 1}

A pilot study using a computer-controlled CRT display failed to confirm the broad findings of previous studies, viz. that ISI is the principal determinant and that the figures are relatively unimportant. The experiment was rerun on a tachistoscope because apparent motion studies are traditionally performed with a tachistoscope and Kolers (Note 1) suggested that the differences in the way the stimuli are produced might affect the results. However, there was no important difference between the two sets of data, so only the data from the tachistoscopic experiment will be presented.

\section{Method}

Apparatus. A Gerbrands Harvard tachistoscope, Model T-4A, was used. Each of the four channels was independently controlled by a Gerbrands 300 series millisecond timer.

Stimuli. The basic shape used was adapted from Kolers and Pomerantz (1971). It is a right-angled trapezoid and occupies an area of about $2^{\circ} \times 1^{\circ}$ of visual angle. This shape was then reflected through a horizontal or vertical axis to form the apparent motion pairs (see Figure 1). The stimulus configuration was such that each stimulus pair produced a characteristic direction of motion which was used to label the conditions. The figures, with 1-mm-thick contours, were drawn with black ink on white card. The background brightness was about $.7 \mathrm{~cd} / \mathrm{m}^{2}$ (as measured by

up

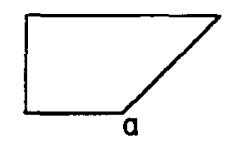

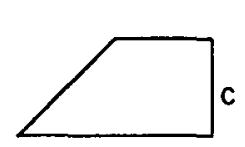

left

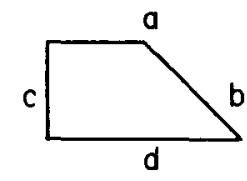

d

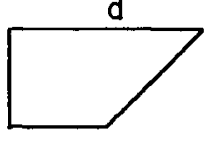

down

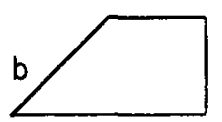

right
Figure 1. The relative sizes and positions of shapes used in Experiment 1. The central shape always appeared first and in the same position, followed by one of the surrounding shapes in its respective location. The four different stimulus configurations are labeled by the direction of the apparent motion that they produced. The contours marked $a-a, b-b, c-c$, and $d-d$ are referred to as "adjacent" sides. an S.E.I. photometer), so the contrast was about .7, where contrast is defined as Contrast $=I(\max )-I(\min ) / I(\max )+I(\min )$.

Subjects. The subjects were undergraduates from Sydney University who participated in the experiment for some nominal course credit.

Procedure. There were 12 different conditions; each condition was specified by one of four different stimulus configurations and one of three different ISIs. The stimulus duration was always $200 \mathrm{msec}$, and the three different ISIs were 25,100 , and $200 \mathrm{msec}$.

Each subject received a block of 20 trials for each of the 12 conditions. At the beginning of each block of trials, both figures were presented simultaneously so that the subjects would know what stimuli to expect. They were then presented with a single cycle of motion (two shapes separated by an ISI) followed by a 3-sec intercycle interval (ICI). During the ICI, they made their responses verbally by calling out "three," "two," or "nothing", to indicate three-dimensional, two-dimensional, or no motion, respectively, and the results were recorded manually. If a subject failed to make a response within the $3 \mathrm{sec}$, that trial was discarded. The order of conditions was randomized across subjects.

The percepts corresponding to the three responses they could make were carefully explained to the subjects before the experiment, and examples were used for illustration. The subjects were discouraged from reporting "no motion" if they were unsure: this response was only to be made if there was no illusion of movement.

Analysis. The important issue in this study is not whether the subject reports any motion, but, given that he does report motion, whether it was three-dimensional or two-dimensional motion. The following procedure was adopted in order to provide an index of how the ambiguity was resolved. Let $R R$ (for rigid rotation) be the number of trials where three-dimensional motion was reported and PD (for plastic deformation) be the number of trials where two-dimensional motion was reported. For every subject under every condition, the value of $\mathrm{PD} /(\mathrm{RR}+\mathrm{PD})$ was calculated. This proportion gives a direct index of how a subject resolves the ambiguity in a given condition. A high value indicates that he mainly saw two-dimensional motion, whereas a low value indicates that he mainly saw three-dimensional motion. It also has the effect of normalizing the data from all subjects, which has the minor drawback of not allowing one to differentiate between subjects who could see motion easily and those who could not. These proportions were placed in a Subjects by Conditions matrix, which was then analyzed by a Friedman two-way analysis of variance by ranks (Ferguson, 1971, chap. 22).

\section{Results and Discussion}

The average values of $\mathrm{PD} /(\mathrm{RR}+\mathrm{PD})$ for 48 subjects are shown in Table 1 . The effect of stimulus configuration (direction) collapsed across ISIs was found to be significant $\left(\chi^{2}=10.29,3 \mathrm{df}, \mathrm{p}<.05\right)$. The effect of stimulus configuration for the $25-\mathrm{msec}$ ISI $\left(\chi^{2}=10.77,3 \mathrm{df}, \mathrm{p}<.05\right)$ and the 100 -msec ISI $\left(\chi^{2}=18.18,3 \mathrm{df}, \mathrm{p}<.05\right)$ was also significant. The effect of ISI was only significant for the left $\alpha^{2}=$ $7.72,2 \mathrm{df}, \mathrm{p}<.05)$ and right $\left(\chi^{2}=6.28\right.$, $2 \mathrm{df}, \mathrm{p}<.05)$ stimulus configurations. In general, subjects reported the least three-dimensional motion for the right condition and the most for the down condition.

Under the conditions of this experiment, subjects' reports of two-dimensional or three-dimensional motion do not depend on the temporal parameters of the apparent motion display. This is shown by the failure to find any consistent, significant variation 
Table 1

Mean Values of PD/(RR + PD) for Experiment 1

\begin{tabular}{lcccc}
\hline & \multicolumn{3}{c}{ ISI (in Milliseconds) } & \\
\cline { 2 - 5 } Shape & 25 & 100 & 200 & $\chi^{2}$ \\
\hline Right & .30 & .33 & .31 & $6.28^{*}$ \\
Left & .33 & .36 & .36 & $7.72^{*}$ \\
Up & .36 & .25 & .40 & 3.79 \\
Down & .62 & .50 & .49 & 1.14 \\
$\mathrm{x}^{2}$ & $10.77^{*}$ & $18.18^{*}$ & 6.62 & \\
\hline
\end{tabular}

Note-Effect of direction over all ISIs was $\chi^{2}(3)=10.29$. $^{*}$ Effect of ISI over all directions was $\chi^{2}(2)=.70$. Values of chi square were calculated for each row and column as well as the values for the data collapsed across rows and columns. Note that in this and the following tables, the chi square values are calculated on ranks rather than proportions.

${ }^{*} p<.05$.

in responses that can be attributed to the changes in the ISI and by the observation that there do not appear to be any differences between tachistoscopic and computer-driven displays. The temporal properties of each type of display in terms of rise times, decay times, and refresh rates are considerably different, but the similarity of the results suggests that these properties are not major determinants of DAAM.

This experiment suggests that the overall stimulus configuration (i.e., the pattern part of the apparent motion presentation) is important in determining a subject's responses. This is supported by the finding that subjects significantly change their responses as the stimulus configuration is varied and that there is little difference between the tachistoscopic and computer-driven displays despite the fact that the polarity of contrast is reversed. Pantle and Picciano (1976) suggest that a system which is sensitive to the pattern properties of a stimulus will tend to ignore reversals in contrast. The effect of the stimulus configuration seems to be tied to the length of the parallel element in the "adjacent" sides of the two shapes (see Figure 1). Downwards movement, which provided the longest "adjacent" parallel elements, always gave the most three-dimensional motion, whereas rightwards motion, which provided no parallel element, always gave the least.

In this experiment, subjects received blocks of trials where each trial was exactly the same. Kolers and Pomerantz also adopted the same procedure. In an earlier study (White, 1977), it was found that this type of presentation can lead to subject "set." Data collected from this type of presentation tended to show sequential dependencies in that on a given trial the probability that a subject will make a different response to that made on the preceding trial is higher at the beginning of a block of trials than it is at the end of that block. Randomizing the variables so that it is unlikely that any trial is identical to the one preceding it reduces the sequential dependencies but does not eliminate them entirely. The effect of this "set" is to overemphasize the particular features of the display that the subject uses in his decision to respond three-dimensional or two-dimensional motion. It should not affect the ordering of conditions, only their absolute values, so the findings reported here should remain unaffected.

Kolers and Pomerantz (1971) concluded that depthambiguous apparent motion was resolved by temporal characteristics of the stimuli and that the pattern content had no significant effect. The evidence from this experiment questions that conclusion and the problem arises of how to reconcile these differences. There are many differences between the two studies which could account for the different results; for example, Kolers and Pomerantz used trained, as opposed to naive, subjects and there is a difference between the two analysis methods. One particularly important difference is that when Kolers and Pomerantz were examining the depth ambiguity of various stimulus configurations they superimposed the two shapes spatially rather than separating them out as in this experiment. It is possible that superimposing the shapes results in contour masking which might result in underestimating the importance of the stimulus configuration in determining which type of motion is seen. Contour masking would have been minimal in the present experiment with separated shapes.

It is possible that there are subtle interactions which involve ISI but are not apparent in this study. Any such interactions may be hidden by the relatively small number of ISIs and analysis technique used. Furthermore, this particular experiment confounds stimulus configuration with direction of motion. There is some evidence which suggests that phi motion, at least, is qualitatively different when seen in the vertical plane than when seen in the horizontal plane (e.g., Lane \& Horne, 1964).

\section{EXPERIMENT 2}

This experiment was designed to settle the question of whether the results of the previous experiment were due to the direction of motion of the stimuli or to the stimulus configuration. This was done by designing the experiment so that each stimulus configuration was presented at each of the four directions of motion. The CRT display was used for all of the following experiments.

\footnotetext{
Method

Apparatus. The figures were generated by a PDP-8 computer on the face of an HP 1317 display monitor. The computer was also used to control the presentation and timing of the displays. The display monitor used a P4 phosphor which fluoresces with a blue-white color. Subjects viewed the display in a darkened room and recorded their responses by pushing one of a panel of three buttons. Viewing distance was $.50 \mathrm{~m}$ and was maintained by the use of a chinrest.
} 
The timing of the apparent motion display caused some concern. The computer program was accurate to better than $1 \%$, but the actual display time depends on the screen phosphor. The decay time of the phosphor is nominally $470 \mu \mathrm{sec}$ to $.1 \%$ of initial brightness. In practice, it was not possible to obtain reliable measures of the decay time, which limits the accuracy that can be ascribed to the timing of the apparent motion display. The practical solution adopted was to set the intensity of the display to a level that was easily visible but which did not produce visible afterimages on the screen. The effect of any phosphor persistence will be to lengthen the stimulus durations and shorten the interstimulus intervals. Since this error will be equal for all conditions, and variation across conditions is the only question of interest, the problem of persistence should have little effect on the results.

Stimuli. The stimuli are illustrated in Figure 2, which shows the stimuli as they appeared on the screen. Each condition is labeled by the direction of motion it produces and by the relationship between the closest two sides. Thus, half parallel/down denotes the condition where the direction of motion was downwards and the stimuli were such that the closest sides were parallel for part of their length.

Procedure. Two groups of subjects were used: one group of 26 subjects received stimuli with a 25 -msec ISI, and the other group of 25 subjects received stimuli with a 60 -msec ISI. In all cases, the stimulus duration was $200 \mathrm{msec}$. A program error resulted in the 60 -msec group receiving only 15 of the 16 conditions: they did not see the long parallel/up condition, whereas they had two presentations of the half parallel/down condition.

Every subject received 16 blocks of 20 trials with each block containing a single stimulus configuration. At the beginning of

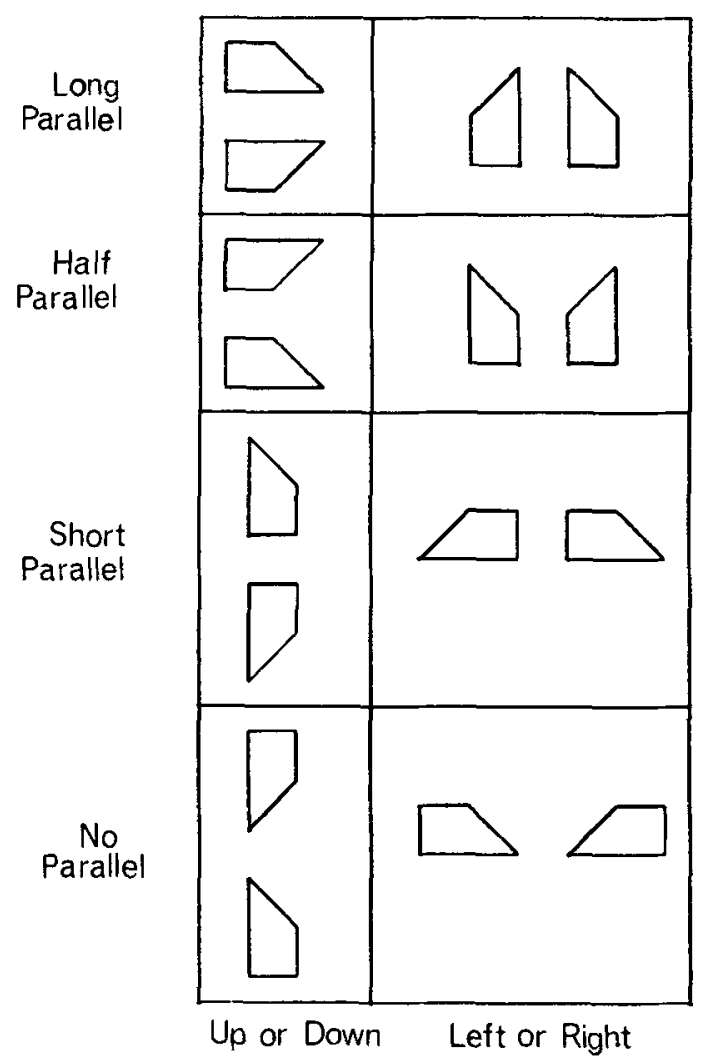

Figure 2. The relative sizes and positions of shapes used in Experiment 2. The shape which appeared first determined the direction of motion.
Table 2

Mean Values of PD/(PD + RR) for Experiment 2

\begin{tabular}{|c|c|c|c|c|}
\hline \multirow[b]{2}{*}{ Shape } & \multicolumn{4}{|c|}{ Parallel Element } \\
\hline & Long & Short & Half & None \\
\hline & \multicolumn{4}{|c|}{$\mathrm{ISI}=25 \mathrm{msec}$} \\
\hline Right & .36 & .51 & .41 & .65 \\
\hline Left & .24 & .38 & .37 & .63 \\
\hline Up & .36 & .51 & .41 & .65 \\
\hline \multirow[t]{2}{*}{ Down } & .22 & .29 & .37 & .51 \\
\hline & \multicolumn{4}{|c|}{ ISI $=60 \mathrm{msec}$} \\
\hline Right & .36 & .32 & .44 & .40 \\
\hline Left & .29 & .32 & .33 & .42 \\
\hline Up & & .41 & .30 & .58 \\
\hline Down & .21 & .32 & .32 & .58 \\
\hline
\end{tabular}

Note-Effect of shape across directions was $\mathrm{x}^{2}(3)=16.08$ $(p<.05)$ for $25 \mathrm{msec}$ and $\mathrm{x}^{2}(3)=13.84(p<.05)$ for $60 \mathrm{msec}$. Effect of direction across shapes was $x^{2}(3)=7.58$ for $25 \mathrm{msec}$ and $x^{2}(3)=2.46$ for 60 msec. There were no data for the cell left blank. Chi square values were calculated only on the collapsed data. (See Figure 2 for an illustration of the stimuli.)

each block, a static display (such as in Figure 2) appeared on the screen for $2 \mathrm{sec}$, which indicated to the subject the stimuli that would be used in the coming block. It was followed by a 500 -msec blank and then the first trial. The order of conditions was randomized across subjects.

\section{Results and Discussion}

The data from this experiment were analyzed in two separate ways. In the first case, the data were collapsed across direction of motion and tested for the effect of shape. In the second case, the data were collapsed across shape and tested for the effect of direction of motion. As in the last experiment, these analyses were done by calculating a value for $\mathrm{PD} /$ (RR + PD) for each subject under each condition and then analyzing the resultant matrix with the Friedman two-way analysis of variance by ranks.

In both ISI groups, the effect due to shape was significant, whereas the effect due to direction of motion was not (see Table 2). In both ISI conditions, the ordering of the stimulus configurations in terms of increasing three-dimensional responses was long parallel, half parallel, short parallel, and no parallel. The results of this experiment indicate that a subject's response of two- or three-dimensional motion is independent of the direction of motion of the stimuli. They also confirm that the stimulus configuration has a marked effect on a subject's response.

\section{EXPERIMENT 3}

This study draws heavily on Experiment 2 in Kolers and Pomerantz' paper. There are two principal differences between their stimulus conditions and those used in this study: they used three different transformations of the base stimulus and they spatially superimposed the two shapes. In their Experiment 3, 

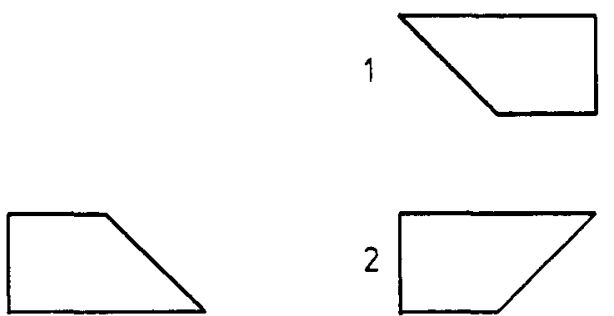

2

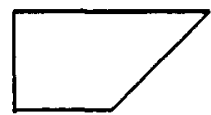

3

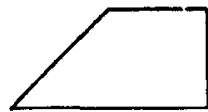

Figure 3. The stimuli used in Experiment 3. The shape on the left always appeared first, followed by one of the shapes on the right. Both figures appeared in the same spatial location. The condition code numbers are shown.

Kolers and Pomerantz used spatially disparate stimuli but they did not examine what effect this had on the depth ambiguity of the display, only the effect it had on the probability of seeing motion. The aim of this experiment was to find out what effect superimposition of the figures has on the resolution of the ambiguity.

\section{Method}

Stimuli. The stimuli are illustrated in Figure 3. The shape on the left was presented first followed by one of the shapes on the right in the same spatial location, i.e., superimposed.

Procedure. Every subject received 12 blocks of 30 trials. Within each block of trials, the shapes were always the same and just one of the four possible ISIs $(25,50,100$, or $200 \mathrm{msec})$ was used. The order of the blocks was randomized across subjects. At the beginning of each block of trials, the two shapes were presented simultaneously for $1 \mathrm{sec}$ followed by a .5 -sec blank. This was to forewarn the subject about the stimulus shapes.

\section{Results and Discussion}

Forty-one subjects were tested in all, and their data were collapsed in such a way as to give the "main effect" of shape and ISI (see above). The effect due to ISI was found to be nonsignificant $\left(\chi^{2}=6.56\right.$, $3 \mathrm{df}, \mathrm{p}>.05)$. The effect due to shape was also found to be nonsignificant $\left(\chi^{2}=4.4,2 \mathrm{df}, \mathrm{p}>.05\right)$ (see Table 3).

Table 3

Mean Values of PD/(PD + RR) for Experiment 3

\begin{tabular}{ccccc}
\hline & \multicolumn{4}{c}{ ISI (in Milliseconds) } \\
\cline { 2 - 5 } & 25 & 50 & 100 & 200 \\
\hline 1 & .60 & .53 & .46 & .56 \\
2 & .68 & .50 & .24 & .58 \\
3 & .74 & .74 & .65 & .56 \\
\hline
\end{tabular}

Note-Effect of shapes across ISIs was $\chi^{2}(3)=6.56$. Effect of ISI across shapes was $\chi^{2}(2)=4.40$. Note that the effect of shape was not found to be significant in this experiment, the only experiment in which this was the case. (See Figure 3 for an illustration of the stimuli.)
In this experiment, changing the stimulus configuration did not result in a change in the relative proportions of two-dimensional and three-dimensional responses. The failure to find an effect due to the ISI has been a consistent result. Casual observation suggests that two- and three-dimensional motion are perceptually very similar when the stimuli are superimposed. They look much more similar than when the same figures are used in a spatially disparate display, as in the previous experiment.

Direct comparisons between Kolers and Pomerantz' results and these are not possible, not least because they used an entirely different analysis method. However, in their Experiment 2 they found that the effect of ISI was about the same as the effect of stimulus duration and that the effect of stimulus configuration was minimal. It may be that the stimulus configuration chosen by them to test the depth ambiguity happened to be a particularly difficult situation to discriminate and the subject may have relied on other properties of the display for his discrimination. This experiment suggests, however, that if the shape information is unable to provide a basis for discrimination, then the ISI information does not take over its role.

\section{EXPERIMENT 4}

The results of these experiments suggest that the stimulus configuration is, in fact, a significant variable in resolving depth-ambiguous apparent motion. In particular, it appears that the relationship between the two closest sides of the figures is important. The amount of parallel elements in these "adjacent" sides and how close they are to each other both appear to be important variables. This experiment is designed to test two predictions: that for a given separation the length of parallel elements in the adjacent sides of the figures will determine the threedimensional responses and that for a given figure increasing the separation leads to fewer threedimensional responses.

The basis for these predictions is the hypothesis that whether the object will appear to rotate or not depends on the ease with which the axis of rotation can be identified. Parallel adjacent sides close to one another will make the axis of rotation more salient, whereas nonparallel adjacent sides will make it more difficult to identify. The assumption is that DAAM is influenced by complex figural properties to a much greater extent than has been previously supposed.

\section{Method}

Stimuli. The stimuli are illustrated in Figure 4. The first shape always appeared in the same spatial location, but the second shape could appear in any one of seven different spatial locations. The direction of the motion could therefore be either up or down, but this had been found to have no significant effect (see Experiment 2 above). 

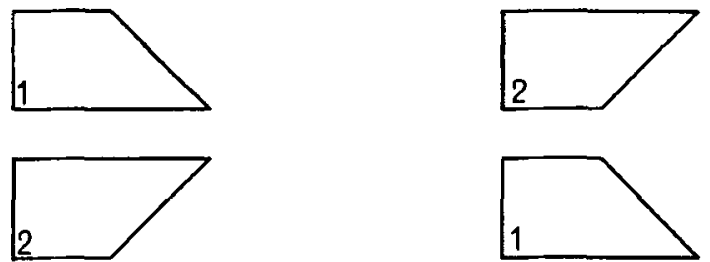

5

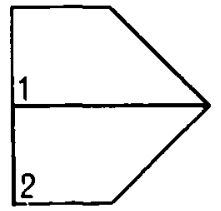

6

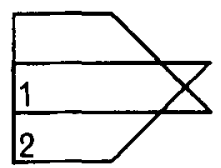

7

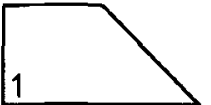

4

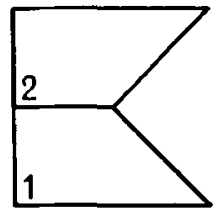

3

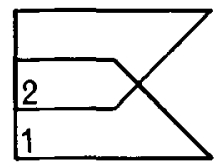

2

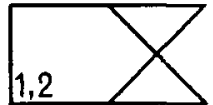

1

Figure 4. The stimuli used in Experiment 4. The shaped labeled "1" always appeared first and in the same spatial location, followed by the shape labeled " 2 ." The condition code number appears beneath each set of figures.

Procedure. Each subject received a single block of 210 trials. Within this block of trials, the subject received 30 presentations of each of the 7 different stimulus configurations and 70 presentations of each of the three ISIs used $(25,100$, and $200 \mathrm{msec})$. The orders of presentation were randomized within and across subjects.

\section{Results and Discussion}

Fifty subjects were tested, and their data were analyzed as in the previous experiments. Collapsing the data across stimulus configuration, the effect of ISI was found to be nonsignificant $\alpha^{2}=2.56,2 \mathrm{df}$, $\mathrm{p}>.05$ ). Collapsing the data across ISI, the effect of stimulus configuration was found to be significant $\left(\chi^{2}=66.07,6 \mathrm{df}, \mathrm{p}<.05\right)$ (see Table 4).

Given the results of the previous experiments, it is not surprising that there was an effect due to stimulus configuration. The important question here is whether the results follow the pattern predicted on the basis of the hypothesis of proximity and length of parallel elements in the adjacent sides. Referring to Figure 4, Condition 1 should rank highest for two-dimensional motion because here the sides of the figure are most distant from each other. Conditions 6 and 3, 7 and 2, and 5 and 4 form pairs, with the two-dimensional response predicted to increase from pair to pair because of the increasing separation. Within each

pair, the first member will rotate more because of the longer parallel element. This information is presented in Figure 5, which shows the predicted and obtained average ranks for each of the stimulus configurations. Using Spearman's coefficient of rank correlation, there is a significant association between the predicted and obtained ranks $(\varrho=.832, \mathrm{p}<.05)$.

Earlier, the hypothesis was discussed that DAAM is resolved by the principle that as phenomenal separation is increased, ISI must also be increased in order to preserve "good" motion. On this hypothesis, rotational movement would occur at longer ISIs than would two-dimensional movement, because rotation involves greater phenomenal movement of the contours of the figures.

In order to derive predictions from this hypothesis, one needs a measure of total contour movement during apparent motion. The easiest case to consider is the three-dimensional percept. Consider the area swept out by the shape as it rotates about an axis from one position to the other, i.e., the surface area of the cylinder of rotation. ${ }^{1}$ It is intuitively obvious that the relation between the surface area of the cylinder of rotation and contour element movement is monotonic. That is, large surface areas imply contour elements moving along large arcs, whereas small surface areas imply movement along short arcs. One can thus rank the stimuli used in this experiment on the basis of these surface areas and one would assume that the smallest areas would yield the most three-dimensional motion. The results of this ranking are plotted in Figure 5, and there is no significant degree of association between this predicted curve and the actual results $(e=.35, \mathrm{p}>.05)$.

The results of this experiment support the hypothesis that, under the conditions of this experiment, the relative proportions of two-dimensional and threedimensional responses can be determined by the properties of shapes. These properties are almost certainly not the sole determinants of the response, nor are the proposed guidelines for determining the rela-

Table 4

Mean Values of PD/(RR + PD) for Experiment 4

\begin{tabular}{cccc}
\hline & \multicolumn{3}{c}{ ISI (in Milliseconds) } \\
\cline { 2 - 4 } Stimulus & 25 & 50 & 100 \\
\hline 1 & .81 & .74 & .73 \\
2 & .61 & .54 & .54 \\
3 & .32 & .31 & .30 \\
4 & .39 & .37 & .40 \\
5 & .39 & .43 & .41 \\
6 & .34 & .32 & .33 \\
7 & .57 & .54 & .49 \\
\hline
\end{tabular}

Note-Effect of stimulus configuration over ISIs was $\chi^{2}(6)=$ $66.07(p<.05)$. Effect of ISI over stimulus configurations was $\mathrm{x}^{2}(2)=2.56$. (See Figure 4 for an illustration of the stimuli.) 


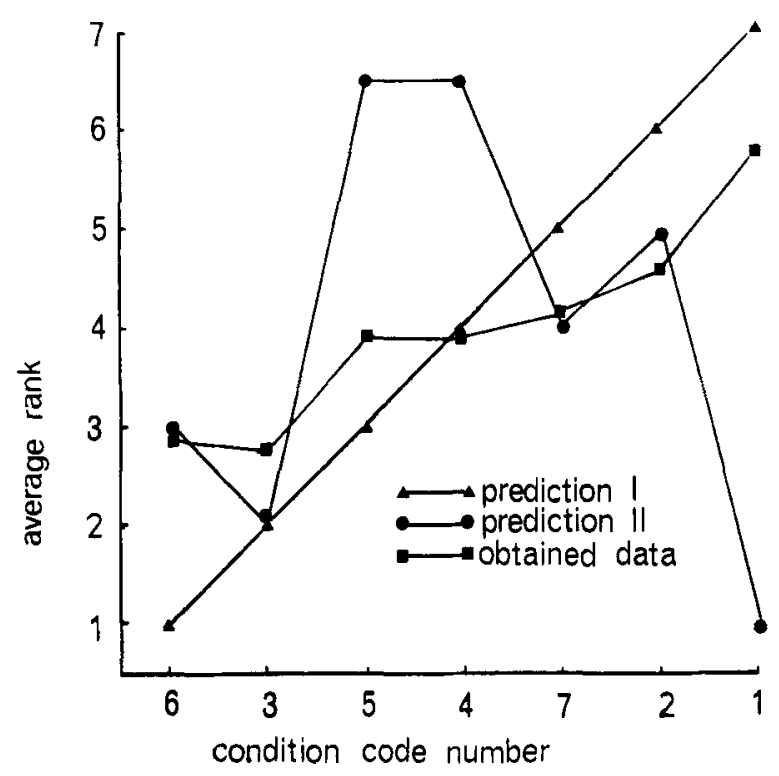

Figure 5. Graphs of the obtained and predicted average ranks for three-dimensional motion for the stimuli illustrated in Figure 4. Prediction $I$ is based on the explanation offered in this study. Prediction $I I$ is based on the explanation based on Korte's third law.

tive proportions likely to be exhaustive. However, it does seem that a complete explanation of the resolution of DAAM must include the effect of the shapes themselves.

\section{GENERAL DISCUSSION}

The results that Kolers and Pomerantz obtained led them to conclude that depth-ambiguous apparent motion was not essentially different from ordinary two-dimensional apparent motion. The two percepts occurred in different temporal ranges, which is consistent with the idea that the critical difference between the two percepts is the phenomenal path traveled by the object. Given that this is the case, then, the pattern properties of the stimulus are relatively unimportant and only affect the phenomenon insofar as they permit two paths. That is, any shape can follow a two-dimensional path, but only certain shapes, such as those to which a direction can be assigned, could logically be expected to follow a three-dimensional path (Kolers, 1972).

The finding that changing the stimulus configuration alters the relative proportions of two-dimensional and three-dimensional responses casts some doubt on this conclusion. It may be that ISI is the principal determinant of depth-ambiguous apparent motion when the figural relationships are made obscure, such as by superimposing the shapes. It may be that there is a subtle interaction between figural properties and ISI, with the effect of each dominating under particular conditions. These experiments were not designed to explore any such interaction, and the analysis technique is not powerful enough to detect them.

The problem remains of where to fit the phenomenon of DAAM in our understanding of the perceptual system. The current tendency has been to think of motion, both real and apparent, as being mediated by an amalgamation of outputs from the sustained and transient systems in the human visual system (e.g., Kulikowski, 1978a, 1978b). There is evidence that the two systems are not independent (e.g., Singer $\&$ Bedworth, 1973), with the activity of the transient channel inhibiting output from the sustained channel. This interaction is reflected in a number of psychophysical results (Breitmeyer \& Ganz, 1976; Breitmeyer, Love, \& Wepman, 1974; von Grunau, 1978) where the presence of movement raises the threshold for detecting the pattern of the stimulus.

When studying the interaction between the sustained and transient channels, the emphasis has been on threshold phenomena. DAAM does not appear to be a threshold phenomenon in that the pattern is above threshold and the subject clearly sees an object moving in one of two different modes. DAAM is not simply a matter of detection, since the question of which type of motion was seen arises only when there is perceived motion. Rather, it is a complex, suprathreshold phenomenon where the incidence of a particular visual experience is facilitated or inhibited by the spatial pattern of the stimulation.

DAAM is probably very much under the control of higher "cognitive" processes in that the shape is made to rotate when the images received are consistent with the percept of a single object rotating in depth. The pattern detection properties of the sustained channel are relevant insofar as they determine the quality of the information available to these cognitive centers. One can assume that in an apparent motion situation the form information is not complete, that there are deletions and distortions in the shapes due to the time constant of the channel and the inhibiting effect of the transient channel. The exact amount and type of information available would probably vary randomly over presentations. The form information from both presentations would then be assessed to determine if it fits with a rotation paradigm, such as the presence of symmetry about an axis or the definition of an axis of rotation. Clearly, some pattern configurations can be degraded more than others and still retain this essential information. Such configurations will, on average, yield more threedimensional responses. If the form information is so degraded as to be inconsistent with a rigid shape rotating, then the resulting ill-defined form information is coupled with the motion information to form the concept of plastic, two-dimensional motion. This 
model assumes that rigid shapes rotating in depth will be the preferred concept, since it is consistent with past experience.

The processes which generate DAAM are still not understood, but this study has shown that the phenomenon should be removed from the realm of simple apparent motion where it was placed when it was thought that the only important determinants of the phenomenon were the temporal properties of the stimulus. The demonstration that the figural properties are at least as important means that depthambiguous apparent motion may be best understood as a special type of ambiguous pattern. An understanding of the phenomenon is likely to lie in an understanding of the processes which govern the perception of other ambiguous patterns.

\section{REFERENCE NOTE}

1. Kolers, P. A. Personal communication, 1977.

\section{REFERENCES}

Attneave, F. Apparent motion and the what-where connection. Psychologia, 1974, 17, 108-120.

Breitmeyer, B. G., \& Ganz, L. Implications of sustained and transient channels for theories of visual pattern masking, saccadic suppression and information processing. Psychological Review, 1976, 83, 1-36.

Breitmeyen, B. G., Love, R., \& Wepman, B. Contour suppression during stroboscopic motion and metacontrast. Vision Research, 1974, 14, 1451-1456.

Ferguson, G. A. Statistical analysis in psychology and education (3rd ed.). New York: McGraw-Hill, 1971.
Kole rs, P. A. Aspects of motion perception. Oxford: Pergamon Press, 1972.

Kolers, P. A., \& Pomerantz, J. R. Figural change in apparent motion. Journal of Experimental Psychology, 1971, 87, 99-108.

Kulikowski, J. J. Pattern and movement detection in man and rabbit: Separation and comparison of occipital potentials. Vision Research, 1978, 18, 183-190. (a)

Kulıkowski, J. J. Spatial resolution for the detection of pattern and movement (real and apparent). Vision Research, 1978, 18, 237-238. (b)

Lane, W. P., \& Horne, E. P. Some evidence that vertical motion is easier than other directions. Journal of General Psychology, 1964, 71, 87-92.

Orlansky, J. The effect of similarity and difference in form on apparent movement. Archives of Psychology, 1940, 85, 246.

Pantle, A., \& Picciano, L. A multistable movement display: Evidence for two separate motion systems in human vision. Science, 1976, 193, 500-502.

Shepard, R. N., \& Judd, S. A. Perceptual illusion of rotation of three-dimensional objects. Science, 1976, 191, 952-954.

Singer, W., \& BEDWORTH, N. Inhibitory interaction between $\mathrm{X}$ and $\mathrm{Y}$ units in the cat lateral geniculate nucleus. Brain Research, $1973,49,291-307$.

von Grunau, M. W. Interaction between sustained and transient channels: Form inhibits motion in the human visual system. Vision Research, 1978, 18, 197-201.

Whiте, D. G. Factors influencing the perception of depth ambiguous apparent motion. Unpublished MSc thesis, Sydney University, 1977.

\section{NOTE}

1. This assumption weights both the length of the element and the distance it travels. Predictions derived from considering only the distance traveled are slightly different but similarly inconsistent with the experimental data.

(Received for publication January 8, 1979; revision accepted August 10, 1979.) 